\title{
A BRIEF STUDY TO CLARIFY SOME ASPECTS RELATED TO VIBRATIONAL DENSITY OF STATES FOR THE FAR INFRARED RANGE IN AMORPHOUS SEMICONDUCTORS
}

\author{
M. A. GRADO-CAFFARO and M. GRADO-CAFFARO
}

C/Julio Palacios 11, 9으 28029-Madrid, Spain

(Received 23 May 1997; In final form 22 July 1997)

\begin{abstract}
Phonon density of states of amorphous semiconductors for the far-infrared range is examined analytically. On the basis of this formulation, optical absorption corresponding to structural disorder is evaluated and discussed at the far-infrared range for a-Ge and $\mathrm{a}-\mathrm{Si}$.
\end{abstract}

Keywords: Phonon density of states; amorphous semiconductors; far-infrared range; structural disorder

\section{INTRODUCTION}

Vibrational (or phonon) density of states of amorphous tetrahedrally bonded semiconductors plays an important role in order to examine optical absorption corresponding to structural disorder. On the other hand, the relation between the vibrational density of states and the relaxation of the wave-vector conservation rule plays also an important role but the relaxation of this rule is not crucial in explaining the basic optical properties of the above solids [1], [2].

Germanium and silicon are good candidates for studying vibrational excitations (phonons) in the disordered phase. Although various basic aspects related to these excitations are relatively well understood, there are some problems which remain unsolved from the 
theoretical point of view with respect to the major part of amorphous semiconductors; in particular, we can mention a-Ge and a-Si as typical elements of group IV; these solids will be the subject of the following exposition.

\section{THEORETICAL CONSIDERATIONS}

First, we consider the following expression for the phonon density of states of amorphous group IV semiconductors in the far-infrared range [3]:

$$
g(\omega)=\frac{1}{N} \sum_{n}^{N}\left|\gamma_{n}\right|^{2} \delta\left(\omega-\omega_{n}\right)
$$

where $\omega_{n}$ is the vibrational eigenfrequency of the $n^{\text {th }}$ normal mode of the solid and $\delta$ denotes Dirac's delta function.

In order to derive Eq. (1), a quasi-harmonic solid has been assumed. In addition, matrix elements $\gamma_{n}$ behave as dependent on experiment and are also model-dependent [4]; this produces a degree of uncertainty which may be overcome by means of deep and rigorous studies that, unfortunately, have not appeared in the literature. Now we consider the structural disorder contribution to the low region of the far-infrared spectrum of a-Ge and a-Si namely [2], [5], [6]:

$$
\alpha^{(s)}(\omega)=\omega \varepsilon_{2}^{(s)}(\omega) \approx k \omega g(\omega)
$$

where $\alpha^{(s)}(\omega)$ denotes absorption coefficient and $k$ is a positive constant. By combining formulae (1) and (2), we may compute the average optical absorption in the far-infrared range as follows (since $\left.\alpha(\omega)=\alpha^{(s)}(\omega)[6]\right)$

$$
\langle\alpha\rangle=\Omega^{-1} \int_{0}^{\Omega} \alpha(\omega) d \omega=k N^{-1} \Omega^{-1} \sum_{n}^{N}\left|\gamma_{n}\right|^{2} \omega_{n}
$$


We consider a large number of phonons $(N \rightarrow \infty)$ since $\omega$ is very low; then from Eq. (3) and by applying Stolz's citerion we have:

$$
\begin{aligned}
\lim _{N \rightarrow \infty}\langle\alpha\rangle & \approx k \Omega^{-1} \cdot \lim _{N \rightarrow \infty} \frac{\sum_{n}^{N}\left|\gamma_{n}\right|^{2} \omega_{n}-\sum_{n}^{N-1}\left|\gamma_{n}\right|^{2} \omega_{n}}{N-(N-1)} \\
& =k \Omega^{-1} \cdot \lim _{N \rightarrow \infty}\left(\left|\gamma_{N}\right|^{2} \cdot \omega_{N}\right) \leq k\left(\lim _{N \rightarrow \infty}\left|\gamma_{N}\right|\right)^{2}
\end{aligned}
$$

because $\omega_{N} \leq \Omega$. Notice that the series $\sum_{n}^{\infty}\left|\gamma_{n}\right|^{2} \omega_{n}$ is divergent since if it were convergent then $\langle\alpha\rangle \rightarrow 0$ which is physically absurd. Therefore, $\lim _{N \rightarrow \infty}\left(\left|\gamma_{N}\right|^{2} \cdot \omega_{N}\right) \neq 0$ as expected. On the other hand, in practice we can take $\Omega \approx 100 \mathrm{~cm}^{-1}$.

\section{CONCLUSIONS}

We have found that the average value of the optical absorption coefficient of a-Ge and a-Si for $\omega \leq \Omega\left(\Omega \approx 100 \mathrm{~cm}^{-1}\right)$ is mainly determined by the limit of the sequence $\left\{\left|\gamma_{N}\right|\right\}$. In addition, $\langle\alpha\rangle$ is strongly related to the sum rule which, as it is well-known, plays an important role in the questions associated with optical absorption. Finally, note the role played by the eigenvalue sequence $\left\{\omega_{N}\right\}$ in the sum rule.

\section{References}

[1] Brodsky, M. H. and Lurio, A. (1972). Bull. Am. Phys. Soc., 17, 322.

[2] Grado-Caffaro, M. A. and Grado-Caffaro, M. (1992). Phys. Lett. A., 169, $399-401$.

[3] Ladan, F. R. and Zylbersztejn, A. (1972). Phys. Rev. Lett., 28, 1198.

[4] Alben, R., Weaire, D., Smith, J. E. and Brodsky, M. H. (1973). Phys. Rev. Lett., 30, 1141.

[5] Grado-Caffaro, M. A. and Grado-Caffaro, M. (1994). Mod. Phys. Lett. B., 8, 247-250.

[6] Mitra, S. S., Paul, D. K., Tsay, Y. F. and Bendow, B. (1974). AIP Conf. Proc. No. 20, 284-289. 

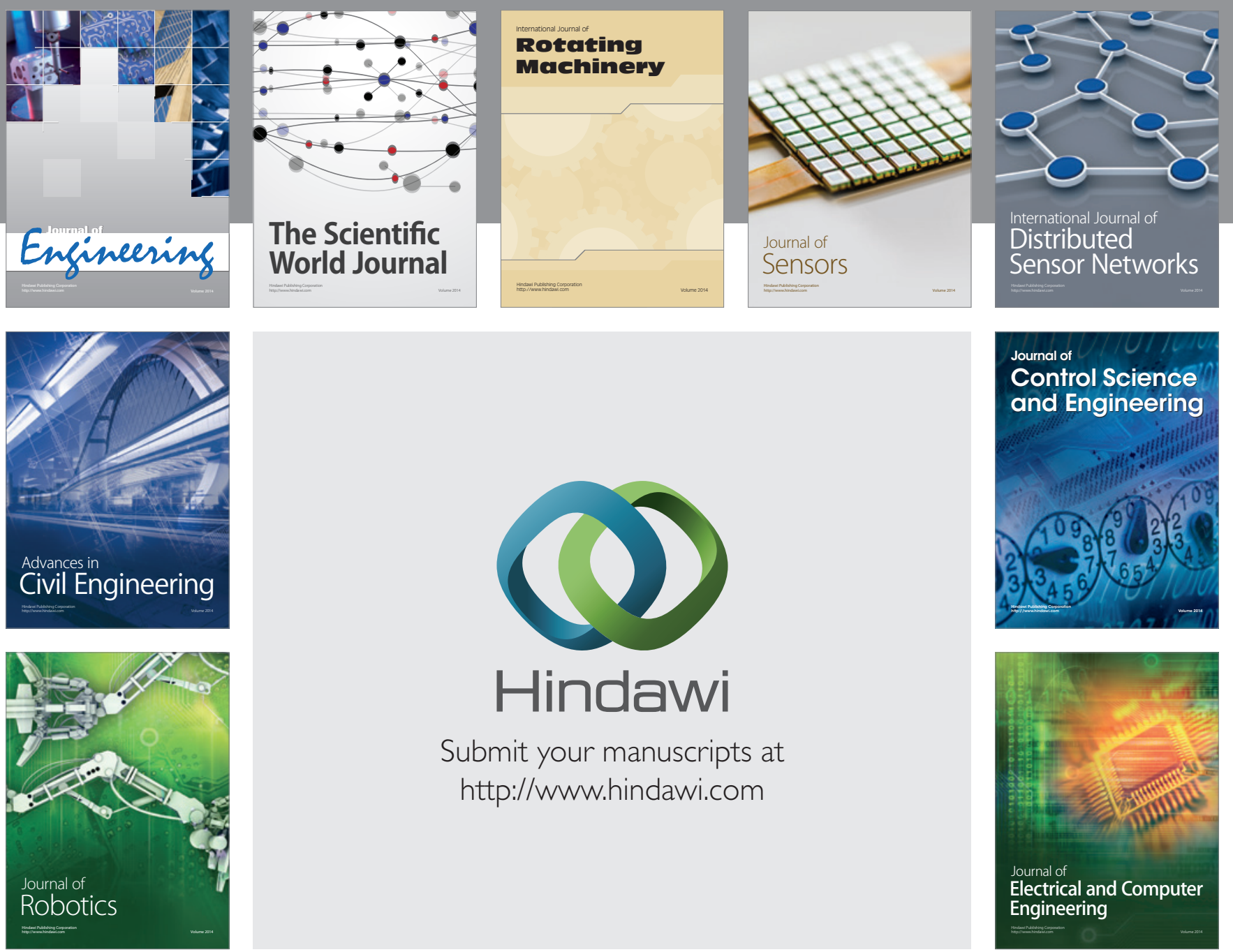

Submit your manuscripts at

http://www.hindawi.com
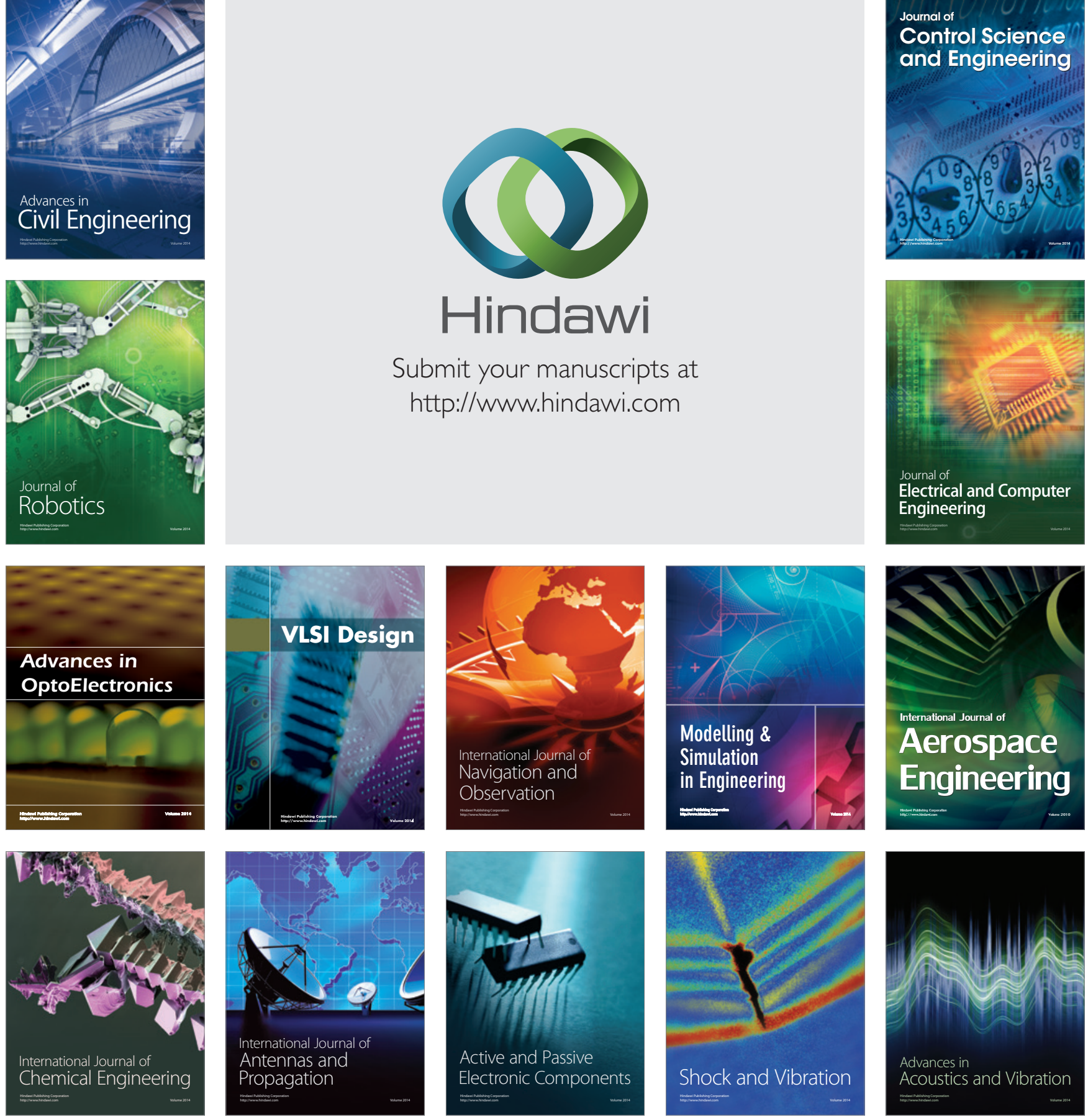